\title{
GaN-based SSD structure for THz applications
}

\author{
Manvi Agrawal \\ School of Electrical and \\ Electronic Engineering \\ Nanyang Technological University \\ Singapore \\ magrawal@ntu.edu.sg \\ Guillaume Ducournau \\ IEMN \\ University Lille 1 \\ Villeneuve D'Ascq, France \\ guillaume.ducournau@univ-lille.fr
}

\author{
Dharmarasu Nethaji \\ TemasekLaboratories@NTU \\ Nanyang Technological University \\ Singapore \\ dharma@ntu.edu.sg \\ Marie Lesecq \\ IEMN \\ University Lille \\ Villeneuve D'Ascq, France \\ marie.lesecq@univ-lille.fr
}

\author{
Radhakrishnan K \\ School of Electrical and \\ Electronic Engineering \\ Nanyang Technological University \\ Singapore \\ eradha@ntu.edu.sg \\ Javier Mateos \\ Department of Applied Physics \\ Universidad de Salamanca \\ Salamanca, Spain \\ javierm@usal.es
}

\author{
Christophe Gaquiere \\ IEMN \\ University Lille 1 \\ Villeneuve D'Ascq, France \\ christophe.gaquiere@uniokv- \\ lille.fr
}

\author{
Tomás González \\ Department of Applied Physics \\ Universidad de Salamanca \\ Salamanca, Spain \\ tomasg@usal.es
}

\author{
Ignacio Íñiguez-de-la-Torre \\ Department of Applied Physics \\ Universidad de Salamanca \\ Salamanca, Spain \\ indy@usal.es
}

\author{
Sergio García \\ Department of Applied Physics \\ Universidad de Salamanca \\ Salamanca, Spain \\ sergio_gs@usal.es
}

\author{
Susana Pérez \\ Department of Applied Physics \\ Universidad de Salamanca \\ Salamanca, Spain \\ susana@usal.es
}

\begin{abstract}
We report on the growth optimization of GaNbased self switching diode (SSD) structure on SiC, designed using Monte Carlo simulations, for the fabrication of nano-scale SSDs to reach $\mathrm{THz}$ emission as a result of Gunn oscillations. Crack-free epistructure with good epi-characteristics and uniformity on 2inch $\mathrm{SiC}$ substrate was achieved. High carrier density of $2 \times 10^{18}$ $\mathrm{cm}^{-3}$ resulted in a low contact resistance of $0.35 \Omega . \mathrm{mm}$.
\end{abstract}

Keywords—Gunn diode, $\mathrm{THz}, \mathrm{GaN}, \mathrm{SiC}, \mathrm{PA}-\mathrm{MBE}$

\section{INTRODUCTION}

The most common solid-state electronics based $\mathrm{THz}$ devices are obtained using Schottky diodes for the frequency multiplication of fundamental oscillators such as resonant tunneling diodes (RTDs), impact ionization avalanche transittime (IMPATT) diode, microwave multipliers and Gunn diodes. The oscillation frequency of classical GaAs and InP Gunn diodes (based on the transfer of electrons to the upper valleys) can only be extended to around $300 \mathrm{GHz}$ limited by the scattering mechanism and energy relaxation frequencies [1]. The superior properties of GaN-based materials such as wide band-gap, high saturation velocity and high breakdown field make them suitable candidates for high power, high frequency and high-temperature applications [2]. GaN-based Gunn diodes can potentially operate at much higher power than traditional GaAs and InP Gunn diodes at very high frequency, above 300 $\mathrm{GHz}$. However, up to now, no clear observations of continuous Gunn oscillations in GaN devices have been achieved, and the realization of a continuous wave GaN-based Gunn oscillator at $300 \mathrm{GHz}$ and beyond has never been reached before. By means of pulse measurements, just the presence of negative differential resistance which leads to a subsequent breakdown of the devices, has been found [3].

Self-switching diodes (SSDs) produce planar asymmetric non-linear devices and simulations show their potential to create $\mathrm{THz}$ waves as a result of Gunn oscillations $[4,5]$. In this work, we report on the growth optimization of GaN-based SSD structure on SiC (Fig. 1 (a)), designed using Monte Carlo (MC) simulations, for the fabrication of nanoscale SSDs (Fig. 1 (b)) to reach THz emission.

\section{EXPERIMENTAL}

The design rules for the growth of the epistructure was provided by means of MC simulations. The designed epistructure was grown on double side polished semi-insulating (SI) 4H-SiC (001) substrate using plasma assisted molecular beam epitaxy (PA-MBE). The growth was initiated by the insitu removal of the native oxide by exposing the surface of the substrate to three cycles of $\mathrm{Ga}$ deposition and flash-off. Subsequently, $100 \mathrm{~nm}$ AlN nucleation layer was grown at a substrate temperature of $740{ }^{\circ} \mathrm{C}$ followed by $1000 \mathrm{~nm}$ undoped two-step $\mathrm{GaN}$ buffer layer at $710{ }^{\circ} \mathrm{C}$. Initial $\mathrm{GaN}$ with a thickness of $100 \mathrm{~nm}$ was grown at III/ $\mathrm{V}=0.85$ followed by the growth of the remaining GaN under III/V $\geq 1$. Finally, $400 \mathrm{~nm}$ silicon doped $\mathrm{GaN}$ active layer with a carrier concentration of $2 \times 10^{18} \mathrm{~cm}^{-3}$ was grown at the same substrate temperature.
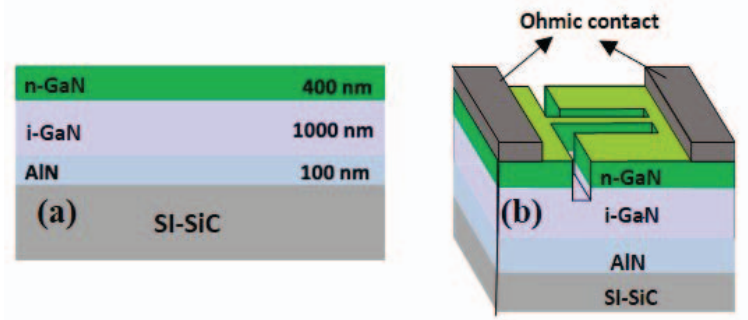

Fig. 1 (a) Cross-sectional schematic of the epistructure and (b) threedimensional geometry of the SSDs.

A set of samples was grown as a function of $\mathrm{Ga} / \mathrm{N}$ (III/V) ratios to achieve good crystalline quality and smooth surface 
morphology of the undoped GaN buffer layer. Moreover, $\mathrm{Si}$ doping was optimized in the subsequent $\mathrm{GaN}$ active layer by varying the temperature of the Si effusion cell.

\section{III.RESULTS}

Fig. 2 (a) and (b) show the AFM images of $5 \times 5 \mu \mathrm{m}^{2}$ scan area for $\mathrm{GaN}$ grown under nitrogen rich conditions (III/V $<1$ ) and metal rich conditions (III/V $\geq 1$ ), respectively. A rough surface morphology (III/V $<1$ ) with RMS roughness of $6.1 \mathrm{~nm}$ while smooth surface (III/V $\geq 1$ ) with an RMS roughness of 0.4 $\mathrm{nm}$ is obtained. Spotty reflection high energy electron diffraction (RHEED) pattern was observed during the growth of $\mathrm{GaN}$ under III/ $\mathrm{V}<1$, indicative of the 3D growth mode while streaky $\mathrm{GaN}$ was obtained under $\mathrm{III} / \mathrm{V} \geq 1$ with a $(2 \times 2)$ reconstruction pattern signifying $2 \mathrm{D}$ growth as shown in the inset of Fig. 2 (a) and (b), respectively.

Fig. 3 shows the $\mathrm{GaN}$ full-width-at-half-maximum (FWHM) along (002) and (102) planes as a function of III/V ratio. For the growth performed in nitrogen rich conditions (III/V < 1.0), the lowest FWHM for GaN (102) was achieved at $\mathrm{III} / \mathrm{V}=0.85$, indicating overall good crystalline quality. In the Ga-stable and Ga rich regions $(1.0<\mathrm{III} / \mathrm{V}$ ratio $<1.2)$, the crystalline quality degraded as the value of FWHM increased while it saturated beyond III/V $>1.2$.

As can be concluded from Fig. 2 and Fig. 3, smooth surface morphology is achieved for growths with III/V $\geq 1$ while good crystalline quality was achieved when III $/ \mathrm{V}<1$. Therefore, a two-step GaN with growth transition from III/V $<1$ to III/V $\geq$ 1 during the initial stage of GaN buffer layer was implemented to obtain reduced dislocation density and smooth surface morphology. Table 1 shows the GaN FWHM along (002) and (102) planes grown using different growth modes.
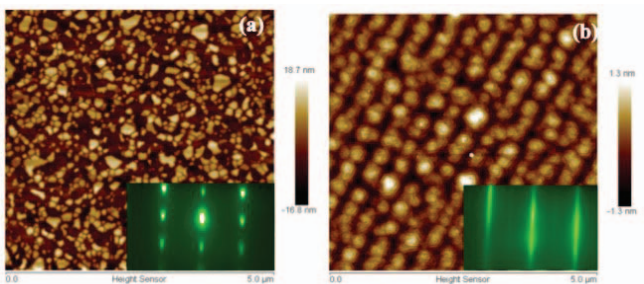

Fig. 2 AFM images of $5 \times 5 \mu \mathrm{m}^{2}$ scan area for GaN grown under (a) III/ $\mathrm{V}<1$ and (b) III/V $\geq 1$. The inset shows RHEED reconstruction patterns of $\mathrm{GaN}$ (a) spotty indicating $3 \mathrm{D}$ growth and (b) streaky $(2 \times 2)$ indicating $2 \mathrm{D}$ growth.

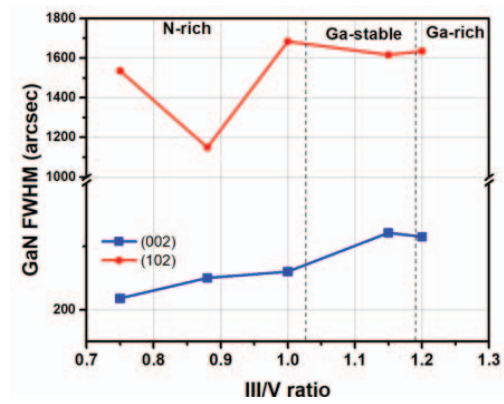

Fig. 3 GaN FWHM along (002) and (102) planes as a function of III/V ratio.
Table 1 GaN FWHM along (002) and (102) planes grown using different growth modes.

\begin{tabular}{|c|c|c|}
\hline \multirow{2}{*}{$\begin{array}{c}\text { Growth } \\
\text { mode }\end{array}$} & \multicolumn{2}{|c|}{ GaN FWHM (arcsec) } \\
\cline { 2 - 3 } & (002) & (102) \\
\hline 3D & 250 & 1150 \\
\hline 2D & 321 & 1616 \\
\hline 3D-2D & 285 & 1207 \\
\hline
\end{tabular}

Fig. 4 shows the carrier concentration in $\mathrm{GaN}$ with respect to different $\mathrm{Si}$ effusion cell temperatures during the growth. As can be seen, a carrier concentration ranging from 0.7 to $6.5 \times 10^{18} \mathrm{~cm}^{-3}$ was obtained. Fig. 5 shows the measured resistance versus the separation between the adjacent contacts of the transmission line measurement (TLM) structures on the epistructure using four-point probe technique. The contact resistance $\left(R_{\mathrm{c}}\right)$ was extracted from the slope and the y-axis intercept of the corresponding linear fit. The TLM was performed on several locations that resulted in an average $R_{\mathrm{c}}$ of $0.35 \Omega . m m$.

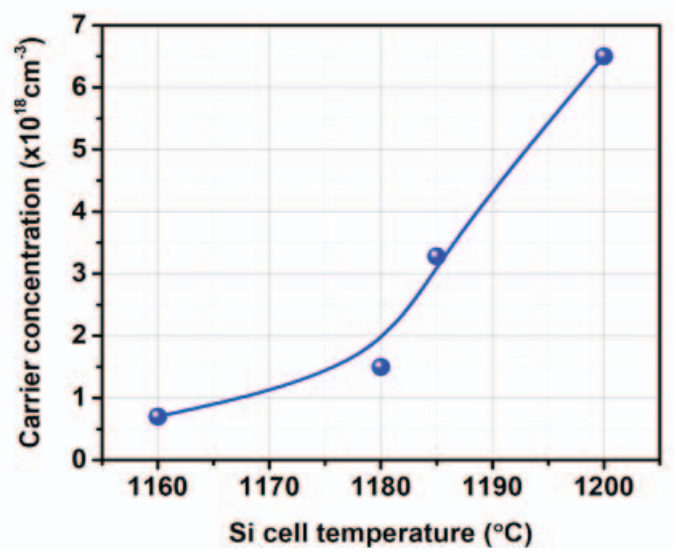

Fig. 4 Carrier concentration in GaN layer with different $\mathrm{Si}$ effusion cell temperature.

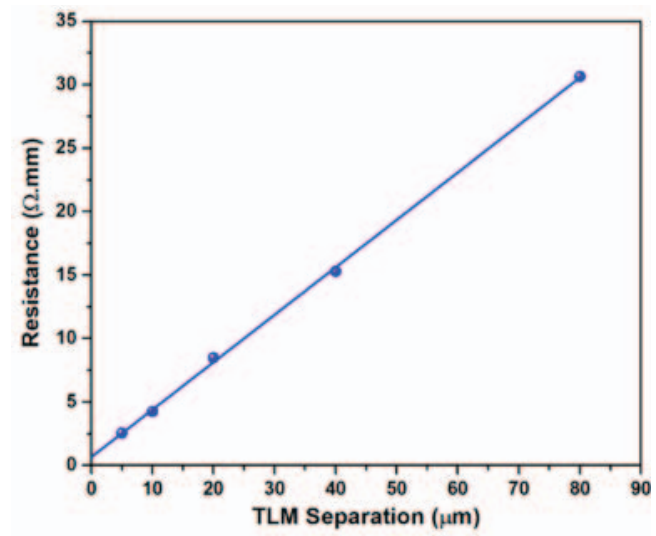

Fig. 5 Resistance versus separation between the adjacent contacts of TLM structures. 
Based on the optimization of the growth conditions for the GaN buffer and the active layers, the complete epistructure was grown on 2-inch $\mathrm{SiC}$ substrate. Fig. 6 (a) shows the photograph of the 2-inch epiwafer and (b) shows the three-dimensional (3D) view of the epiwafer-bow of $19 \mu \mathrm{m}$ obtained by making the measurements in 4 different directions.

Fig. 7 shows the mapping of electrical properties of the epistructure across the 2-inch epiwafer. An average (a) sheet resistance of $390 \Omega / \mathrm{sq}$., (b) mobility of $187 \mathrm{~cm}^{2} /$ V.s (c) and carrier density of $2.14 \times 10^{18} \mathrm{~cm}^{-3}$ are obtained with the standard deviation of $4.2 \%, 6.0 \%$ and $3.2 \%$, respectively. Fig. 8 (a) and (b) show the FWHM mapping along GaN (002) and GaN (102) planes across the epiwafer, and Fig. 9 shows the photoluminescence (PL) mapping of the epistructure, indicating uniform electrical and optical qualities across the wafer.

Table 2 lists the FWHM of GaN along (002) and (102) planes, RMS roughness of $5 \times 5 \mu \mathrm{m}^{2}$ scan area and the average carrier density in the $\mathrm{GaN}$ active layer for the four 2-inch epiwafers. As shown, GaN with improved crystalline quality was obtained with a sub nanometer RMS roughness. The SSD fabrication on these epiwafers is underway and the results will be presented elsewhere.
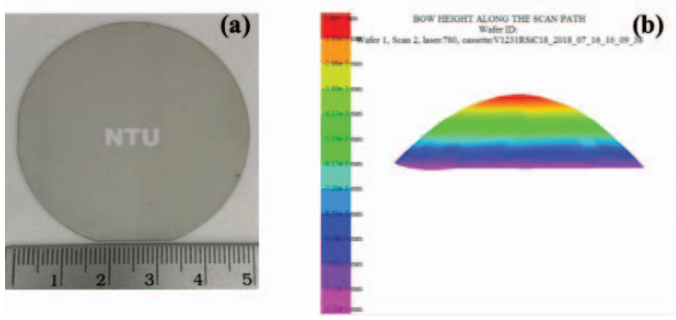

Fig. 6 (a) Photograph of 2-inch epiwafer and (b) the three-dimensional (3D) view of the epiwafer bow.

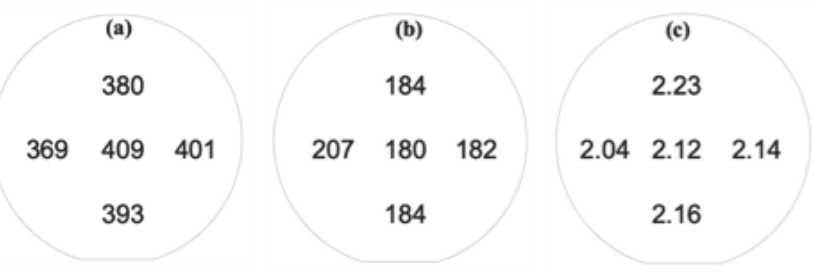

Fig. 7 Electrical properties of the epistructure across the 2-inch epiwafer (a) sheet resistance $\left(\Omega / \mathrm{sq}\right.$.), (b) mobility $\left(\mathrm{cm}^{2} / \mathrm{V} . \mathrm{s}\right)$ and $(\mathrm{c})$ carrier density $\left(\times 10^{18}\right.$ $\left.\mathrm{cm}^{-3}\right)$.
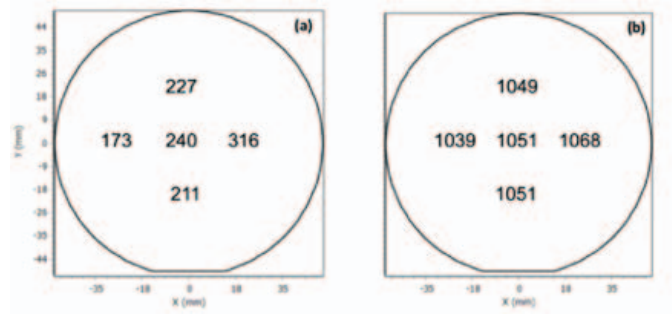

Fig. 8 FWHM mapping along GaN (002) (a) and GaN (102) (b) planes of 2inch epiwafer.

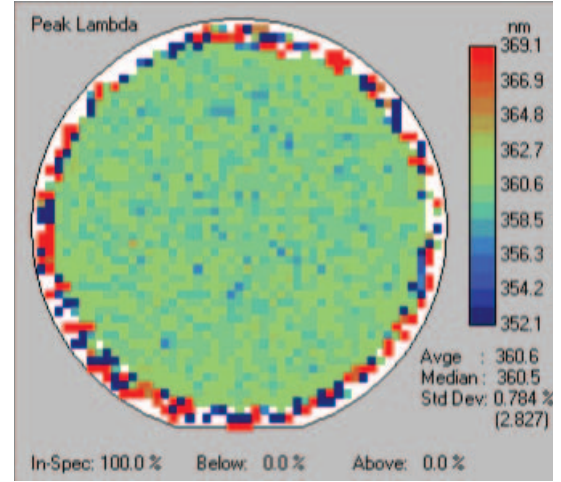

Fig. 9 PL mapping of epistructure.

Table 2 GaN FWHM along (002) and (102) planes, RMS roughness $\left(5 \times 5 \mu \mathrm{m}^{2}\right.$ scan area) and the average carrier density for the four 2-inch epiwafers.

\begin{tabular}{|c|c|c|c|c|}
\hline \multirow[t]{2}{*}{ Wafer ID } & \multicolumn{2}{|c|}{ GaN FWHM(arcsec) } & \multirow[b]{2}{*}{$\begin{array}{c}\text { RSM } \\
\text { roughness } \\
\left(5 \times 5 \mu \mathrm{m}^{2}\right. \\
\text { scan area }) \\
(\mathrm{nm})\end{array}$} & \multirow{2}{*}{$\begin{array}{c}\begin{array}{c}\text { Average } \\
\text { carrier } \\
\text { density }(\times \\
\left.10^{18} \mathrm{~cm}^{-3}\right)\end{array}\end{array}$} \\
\hline & $(002)$ & $(102)$ & & \\
\hline V1231RSiC18 & 250 & 1051 & 0.5 & 2.14 \\
\hline V1260RSiC18 & 240 & 1003 & 0.9 & 2.23 \\
\hline V1261RSiC18 & 246 & 1032 & 0.7 & 2.09 \\
\hline V1273RSiC18 & 297 & 999 & 0.6 & 2.06 \\
\hline
\end{tabular}

\section{IV.SUMMARY}

The GaN-based SSD structure on SiC substrate, designed using $\mathrm{MC}$ simulations was grown by PA-MBE. The crystalline quality of $\mathrm{GaN}$ buffer was improved by using 3D-2D growth modes. High carrier density of $2 \times 10^{18} \mathrm{~cm}^{-3}$ was achieved for GaN active layer with $\mathrm{Si}$ doping and a corresponding contact resistance of $0.35 \Omega . \mathrm{mm}$. Crack-free epistructure with sub nanometer RMS roughness and good uniformity across 2 -inch was established.

\section{ACKNOWLEDGEMENT}

This work has been supported by the NRF2017-NRF-ANR003 GaNGUN project. This work has also been partially supported by the Ministerio de Economía y Competitividad (Spain) and FEDER (European Union) through project TEC2017-83910-R and by the Consejería de Educación de la Junta de Castilla y León (Spain) and FEDER (European Union) through project SA254P18.

\section{REFERENCES}

1. H. Eisele et al., "Submillimeter-Wave InP Gunn Devices," IEEE Trans. Microw. Theory Tech., vol. 52, pp. 2371-2378, October 2004.

2. S.C. Jain et al., "III-nitrides: Growth, characterization, and properties, " J. Appl. Phys., vol. 87, pp. 965-1006, January 2000.

3. N. Ma et al., "Current-controlled negative differential resistance effect induced by Gunn-type instability in n-type GaN epilayers," Appl. Phys. Lett., vol. 96, p. 242104, June 2010.

4. C. Balocco et al., "Microwave Detection at $110 \mathrm{GHz}$ by Nanowires with Broken Symmetry," Nano Lett., vol. 5, pp. 1423-1427, June 2005.

5. O. Drachenko, et al., "Compact magnetospectrometer for pulsed magnets based on infrared quantum cascade lasers," Rev Sci. Instrum., vol. 82, p. 033108, March 2011. 